\title{
ATRACCIÓN DEL TRIPS FRANKLINIELLA OCCIDENTALIS (PERGANDE) (THYSANOPTERA: THRIPIDAE) CON TRAMPAS DE LUZ EN UN CULTIVO DE GERBERA JAMESONII (G.)
}

\author{
ATTRACTION OF THRIPS FRANKLINIELLA OCCIDENTALIS (PERGANDE) \\ (THYSANOPTERA: THRIPIDAE) TO LIGHT TRAPS IN GERBERA JAMESONII \\ (G.) $C R O P S$
}

\author{
Jorge Castresana ${ }^{1}$; Elena Gagliano ${ }^{2}$; Laura Puhl ${ }^{3}$; Silvina Bado ${ }^{4}$; \\ Lourdes Vianna ${ }^{5}$ : Mónica Castresana ${ }^{6}$
}

\begin{abstract}
RESUMEN
El trips de las flores Frankliniella occidentalis (P.) es, sin dudas, uno de los insectos del orden Thysanoptera de mayor peligrosidad en el mundo, ya que produce importantes pérdidas económicas. Esto se debe a su periódica aparición y al daño que ocasiona, tanto por la extracción de savia como por la transmisión de enfermedades virósicas, principalmente en cultivos hortícolas y plantas ornamentales tales como rosa, gerbera y clavel. El monitoreo con trampas adhesivas de color proveen un método simple para estimar la densidad poblacional del trips con poco esfuerzo. Las mismas pueden medir poblaciones de la plaga más fácilmente que los métodos absolutos de monitoreo con uso intensivo de mano de obra, ya que continúan capturando en el tiempo mayor cantidad de trips. La efectividad del uso de trampas adhesivas de color como herramienta para monitoreo de trips ha sido evaluada en invernáculos estando condicionado al atractivo de las trampas y a las tasas de captura de trips según la especie. Al observarse un importante daño de trips, predominantemente en las flores en un cultivo de Gerbera jamesonii ubicado en un invernáculo de producción de flores de corte de la EEA INTA Concordia, provincia de Entre Ríos (Argentina), se inició el presente trabajo cuyo objetivo fue evaluar la efectividad de trampas de luz adhesivas de dos coloraciones (amarillas y azules, ambas seleccionadas por su reconocida atracción a diferentes especies de trips y con diferentes intensidades de luz ( 25 y 40 watts) sobre la captura de trips de las flores. La unidad muestral fue la trampa de luz, mientras que los tratamientos fueron: color de la trampa de luz (amarilla y azul), a dos intensidades distintas ( 25 y 40 watts), siendo por cada tratamiento un total de cuatro repeticiones. Las trampas de luz adhesivas fueron dispuestas en los canteros de Gerbera jamesonii, a razón de cuatro por cantero a los lados de los mismos, a la altura media de las flores de corte. La disposición de las trampas de cada tratamiento en la parcela experimental fue al azar. Las lámparas eran encendidas al atardecer y apagadas por la mañana, momento en que se llevaban al laboratorio con el fin de contabilizar el número de trips adultos capturados. Las trampas de luz amarillas de ambas intensidades ( 25 y 40 watts) resultaron ser significativamente más atractivas para la captura de adultos de $F$. occidentalis en comparación con las de color (azul) e igual intensidad. Por otra parte, las trampas de luz adhesivas indistintamente de su color, tanto amarillo como azul, resultaron ser significativamente más eficientes en la captura del trips $F$. occidentalis cuando las lámparas poseían una intensidad de 40 watts.

Palabras clave: Frankliniella occidentalis, trips, trampas de luz, Gerbera jamesonii.
\end{abstract}

\author{
ABSTRACT \\ Flower thrips Frankliniella occidentalis (P.) may cause severe economic losses due to its periodic occurrence, the damage caused \\ by sucking sap and spreading plant diseases through the transmission of viruses, mainly in horticultural crops and ornamental \\ plants, such as roses, gerbera and carnation. Monitoring through sticky colored traps provides a simple method of obtaining es- \\ timates of thrips population densities. These traps may measure a far more rapid estimate of pest population than labor-intensive \\ 1 Instituto Nacional de Tecnología Agropecuaria (INTA) - Estación Experimental Agropecuaria (EEA). Concordia. E-mail: \\ jcastresana@correo.inta.gov.ar \\ 2 INTA-EEA. Concordia. E-mail: huertacdia@correo.inta.gov.ar \\ 3 Cátedra de Métodos Cuantitativos Aplicados (Facultad de Agronomía Universidad de Buenos Aires - FAUBA). \\ E-mail:1puhl@agro.uba.ar \\ 4 INTA-EEA. Chubut. E-mail: sbado@ chubut.inta.gov.ar \\ 5 INTA-EEA. Concordia. E-mail: vianna@ correo.inta.gov.ar \\ 6 Traductora Pública en Inglés (Universidad de Buenos Aires - UBA) E-mail: moni.castresana@ gmail.com
}

Fecha de Recepción: 01 Agosto 2007

Fecha de Aceptación: 10 Diciembre 2007 
absolute methods of counting individuals, as these traps continuously catch a higher number of thrips throughout the time. The utility of these sticky colored traps methods as tools for monitoring thrips population has been assessed in greenhouses, taking into consideration the traps attractiveness and the capture rate according to species. This work was conducted once a serious damage on flowers (Frankliniella occidentalis) caused by thrips was observed, mainly in a Gerbera jamesonii crop located in a greenhouse for cut flowers production in the Argentine Agricultural Experiment Station (EEA) of the National Institute for Agricultural Technology (INTA) of Concordia, Province of Entre Rios (Argentina). The main purpose of this work was to evaluate the effectiveness of sticky light traps of two colors (yellow and blue, both of them selected due to its known attractiveness to various thrips species and different light intensities (25 and 40 watt bulb)) on the capture of flower thrips. These traps were made from cardboard boxes $(22 \times 30 \times 20 \mathrm{~cm})$ with an opening $(10 \times 15 \mathrm{~cm})$ through which light passes and covered with a plastic wrap coated with adhesive material on which the adults thrips become trapped (The Tanglefoot Company Adhesive Pest Management \& Tree Protection Products, USA). The trial consisted of the following four treatments: (i) yellow light trap with 25 watt bulb; (ii) yellow light trap with 40 watt bulb; (iii) blue light trap with 25 watt bulb and (iv) blue light trap with 40 watts bulb. Each treatment was replicated four times. Four sticky light traps were positioned on both sides of the Gerbera jamesonii flowerbeds to half-height of cut flowers. For each trial, the trap placement in each experimental design was at random. The bulbs were turned on at sunset and turned off in the morning when the traps were removed and carried to the laboratory and the number of adult thrips caught was counted. On the one hand, yellow light traps with both intensities (25 and 40 watt bulbs) became significantly more attractive to F. occidentalis adults in comparison to the capture rate on the blue ones with the same intensity; and, on the other hand, both yellow and blue sticky light traps were much more efficient for the capture of thrips $\mathrm{F}$. occidentalis with 40 watt intensity bulbs. One reason why thrips would respond to various visual stimuli (a more effective response to $w$ color and its high intensity) would be related to its response to the different changes of physiological features of plants. These changes would play a significant important role in sending a visual signal (color and intensity), which would lead Frankliniella occidentalis (P.) to change the search behavior as to its feeding sources and oviposition.

Key words: Frankliniella occidentalis, thrips, light traps, Gerbera jamesonii.

\section{INTRODUCCIÓN}

\section{IMPORTANCIA DE LA PLAGA}

Un insecto $u$ otro organismo constituye una plaga cuando alcanza un nivel de población suficiente para causar pérdidas económicas en el cultivo. Durante la primavera del 2006 (octubre-noviembre) se hallaron importantes daños ocasionados por trips en cultivos de Gerbera jamesonii (G.) ubicados en un invernáculo de floricultura, situado en la EEA INTA Concordia $\left(31^{\circ} 18^{\prime} \mathrm{S}\right)$.

El trips que se encontró en mayor abundancia fue identificado como el "trips occidental de las flores o trips californiano" Frankliniella occidentalis (Pergande), el cual es originario del Sudoeste de EE.UU. (Bryan \& Smith, 1956) y posee una amplia distribución mundial. Su polifagia, sumada a su alto potencial biótico, le permite producir grandes poblaciones de individuos que colonizan distintos cultivos. En Argentina su presencia ha sido identificada en la década pasada (De Santis, 1995), resultando una de las plagas que causan daños graves en flores de corte de Gerbera jamesonii (Bado y Valvarrosa, 1999), así como de otras especies de flores, tanto en invernadero como al aire libre (Tommasini y Maini 1995; Katayama et al., 1997). Los adultos y las larvas de $F$. occidentalis producen severos daños raspando y succionando el fluido de las células que se encuentra en la superficie de los pétalos, originando vetas y decoloraciones visibles (manchas de color gris-plateado), como también la necrosis de los pétalos de las flores, provocando grandes pérdidas económicas por merma de calidad del producto. Este no puede ser comercializable y por ello ésta es considerada una plaga clave en áreas de producción de flores de corte en el mundo (Yudin et al., 1987 CABI/EPPO, 1998).

Por otro lado, el trips de las flores es una de las especies más eficientes en la transmisión del virus de la peste negra del tomate (TSWV, Tomato Spotted Wilt Virus) (Allen \& Matteoni, 1988), el cual afecta principalmente al tomate, pimiento $\mathrm{y}$ especies ornamentales. Las mermas económicas debidas al TSWV en el área de la Plata han sido de $30 \%$ a $40 \%$ para la producción hortícola y hasta $70 \%$ para flores (Carrizo, 1998).

\section{MÉTODOS CONVENCIONALES DE CONTROL DE LA PLAGA}

Actualmente el control del trips F. occidentalis en invernáculo se lleva a cabo por medio de la aplicación de insecticidas químicos, lo cual no sólo eleva el costo de producción sino que también contamina el medio ambiente. El elevado número de generaciones anuales lleva a que los tratamientos químicos se intensifiquen, produciendo severos daños al complejo de enemigos naturales benéficos y conduciendo al rápido desarrollo de resistencia. 
Otro factor que se debe tener en cuenta es la gran dificultad en el control del insecto debido a su comportamiento. En efecto, las larvas se encuentran protegidas en las flores, las prepupas en el suelo, y el adulto tiene una gran movilidad, interfiriendo en forma negativa con la eficiencia del control con productos químicos de síntesis. Otro factor que interviene es que son llevados a grandes distancias por el viento disminuyendo de ese modo también la eficiencia del control químico.

\section{MONITOREO DE LA PLAGA}

La selección de hospederos en insectos fitófagos consiste en la secuencia de respuestas comportamentales a estímulos visuales, mecánicos, gustativos y olfativos asociadas a plantas hospederas como no hospederas (Visser, 1986). El color y el olor se consideran los atractivos secundarios más importantes para los insectos que visitan las flores (Faegri \& van der Piel, 1979).

Muchos trips adultos pueden distinguir entre diferentes colores (Lewis, 1959; Czenz, 1987; Brødsgaard, 1989; Gillespie \& Vernon, 1990).

Los trips polífagos que se alimentan de tejidos finos de las plantas incluyendo flores tienen una respuesta general para los colores amarillo y azul (Kirk, 1984). La utilidad de las trampas de color adhesivas, como herramientas para monitoreo de trips, ha sido evaluada para invernáculos (Brødsgaard, 1989) y su eficacia en los mismos (Gillespie y Vernon, 1990). Utilizadas las mismas en el contexto de un manejo integrado de esta plaga (MIP), para su detección temprana, permiten jugar un papel central en la estrategia del uso eficiente del insecticida, minimizando las aplicaciones en forma calendario, las cuales, en sistemas intensivos bajo invernáculo como en este caso (cultivos ornamentales), son más frecuentes que en otros cultivos agrícolas, cuando no se halla dicha plaga o cuando la misma se encuentra en niveles demasiados altos que superan el Umbral de Daño Económico (UDE) del cultivo, aumentando como fue mencionado anteriormente el riesgo de intoxicación a las personas que aplican el producto químico y produciendo contaminación en el ambiente. Del mismo modo, provoca en el corto y mediano plazo un aumento de la población de la plaga por la pérdida de enemigos naturales (resurgencia) y generación de resistencia a los pesticidas.

Por esto el implemento de un sistema de monitoreo es un aspecto relevante en todo programa de manejo integrado de plagas (MIP) contribuyendo al beneficio económico de los productores, reduciendo el costo de producción por un uso eficiente de insumos, en donde los insecticidas son uno de los ítems más significativos, maximizando la calidad y el rendimiento del cultivo ornamental, obteniendo un sistema productivo ecológicamente sustentable.

Las trampas adhesivas de color ya han sido usadas para monitorear a las poblaciones de $F$. occidentalis (Brødsgaard, 1993, Shipp et al., 1990). Sin embargo, hay diferencia de opiniones entre autores con respecto a qué color es más atractivo para F. occidentalis y cuál es el más eficiente como herramienta de monitoreo (Cabello et al., 1991 Matteson \& Terry, 1992). Para comprender más detalladamente los mecanismos que actúan en el estímulo visual de F. occidentalis frente a trampas de luz y su efecto atrayente, el objetivo de este trabajo fue analizar la respuesta atractiva de $F$. occidentalis (Pergande) en presencia de trampas de luz adhesivas de color (azul y amarillo), con dos intensidades diferentes (25 y 40 watts), para monitoreo en un cultivo de Gerbera jamesonii, recreando diferentes fuentes de colores parecidas a situaciones encontradas en un hábitat natural.

\section{MATERIALES Y MÉTODOS}

El ensayo fue llevado a cabo en el invernáculo de floricultura de la EEA INTA Concordia, provincia de Entre Ríos ( $\left.31^{\circ} 18^{\prime} \mathrm{S}\right)$, el cual es utilizado para la producción de flores de corte, encontrándose en producción cuatro canteros para la flor de corte Gerbera jamesonii. Desde el 19 de octubre hasta el 29 noviembre de 2006 se realizaron los monitoreos diarios mediante trampas de luz. Estas fueron construidas con cajas de cartón de tamaño 22 x 30 x $20 \mathrm{~cm}$, con una abertura de 10 x $15 \mathrm{~cm}$, por la cual se emite la luz, estando la misma cerrada con film plástico recubierto con goma donde los trips adultos quedaban adheridos (The Tanglefoot Company Adhesive pest management \& Tree protection products, USA). Los tratamientos fueron: color de la trampa de luz (amarilla y azul) con longitudes de onda $(590 \mathrm{~nm})$ y $(490 \mathrm{~nm})$ a su vez la intensidad de las mismas (25 y 40 watts), siendo por cada tratamiento un total de cuatro repeticiones; las trampas de luz fueron distribuidas al azar a lo largo del cantero. Las lámparas eran encendidas al 
atardecer y apagadas por la mañana momento en que se llevaban al laboratorio con el fin de contabilizar el número de trips adultos capturados.

Las trampas de luz fueron colocadas a razón de cuatro por cantero a los costados de los mismos, a la altura media de las flores de corte de gerbera. La unidad muestral fue la trampa de luz seleccionando por día un cantero al azar. Las trampas fueron distribuidas al azar por cantero.

Durante el tiempo en que transcurrió el ensayo no se realizó ninguna aplicación de insecticidas.

Para el análisis estadístico primero se realizó la prueba de Shapiro-Wilks, la cual determinó que la variable respuesta " $\mathrm{N}^{\circ}$ de trips" tiene una distribución normal, cumpliéndose el supuesto necesario para realizar el Análisis de Varianza (ANOVA). Posteriormente se realizó el análisis de Varianza con el fin de detectar si existen diferencias significativas entre las medias de los tratamientos, incluyéndose en el modelo el término (intensidad x color) para detectar si estos factores interactúan.

\section{RESULTADOS}

La influencia de las diferentes variables, color de la trampa de luz, la intensidad del color fuente de luz y la interacción de ambas sobre la preferencia del trips $F$. occidentalis son presentados en el Cuadro 1.1. No se encontró un efecto significativo de interacción color x intensidad de luz (Cuadro 1) ( $p=0,2746)$ en la captura de trips, lo cual indica que el efecto del color de la trampa es independiente de la intensidad de luz. El coeficiente de determinación del modelo propuesto $\left(\mathrm{R}^{2}\right)$ mostró que el 97\% de la variabilidad en el número de trips pudo ser explicada por la intensidad de luz y el color de las trampas.

\section{ELECCIÓN DEL TRIPS F. OCCIDENTALIS HACIA EL COLOR DE LUZ EMITIDO POR TRAMPA}

Los ensayos realizados con las trampas de luz sobre el comportamiento de búsqueda de los trips mostraron que los adultos de F. occidentalis fueron significativamente atraídos por el efecto del color de la lámpara (Figura 1; Cuadro 1) ( $\mathrm{p}<0,001)$ hacia las trampas de luz de color amarillo (AM) (Figura 1).

\section{ELECCIÓN DEL TRIPS F. OCCIDENTALIS HACIA LA INTENSIDAD DE LUZ EMITIDA POR TRAMPA}

Se observaron diferencias significativas en la intensidad del color de las trampas de luz (Figura1; Cuadro 1) $(\mathrm{p}<0,001)$. En efecto, los adultos de F. occidentalis fueron significativamente más atraídos cuando la intensidad fue de 40 watts, en comparación con trampas de luz con lámparas de 25 watt en ambas coloraciones (Figura 1).

\section{ELECCIÓN}

No se encontraron diferencias significativas de interacción color $\mathrm{x}$ intensidad (Cuadro 1) $(p=0,2746)$ en la captura de trips.

\section{Análisis de Varianza}

\section{Cuadro 1}

Respuesta de $\boldsymbol{F}$. occidentalis en ensayos con trampas de luz con diferentes variables (color $\mathrm{e}$ intensidad) Análisis de la Varianza (SC Tipo III)

\begin{tabular}{|l|c|c|c|c|c|}
\hline F. V. & \multicolumn{1}{|c|}{ SC } & G I & CM & F & p-valor \\
\hline Color & 223,13 & 1 & 223,13 & 214,11 & $<0,0001$ \\
\hline Intensidad & \multicolumn{1}{c|}{65} & 1 & 65 & 62,38 & $<0,0001$ \\
\hline Cantero (Bloque) & 69,14 & 3 & 23,05 & 22,11 & 0,0002 \\
\hline Color * intensidad & 1,41 & 1 & 1,41 & 1,35 & 0,2746 \\
\hline Error & 9,38 & 9 & 1,04 & & \\
\hline Total & 368,06 & 15 & & & \\
\hline
\end{tabular}

\section{CONCLUSIONES}

Los ensayos realizados mediante trampas de luz adhesivas mostraron que el color amarillo en ambas intensidades, tanto de 25 como de 40 watts, captura mayor cantidad de individuos de F. occidentalis. El amarillo ha sido identificado como el color preferido para atrapar a Scirtothrips spp., en comparación con las trampas de luz de color azul en ambas intensidades, o sea, 25 y 40 watts, las cuales mostraron una baja atracción al trips.

Una razón por la cual el trips respondería a diferentes estímulos visuales (mayor respuesta al color amarillo como a la mayor intensidad del mismo) serían los diferentes cambios en las características fisiológicas de la plantas, las cuales jugarían un rol 


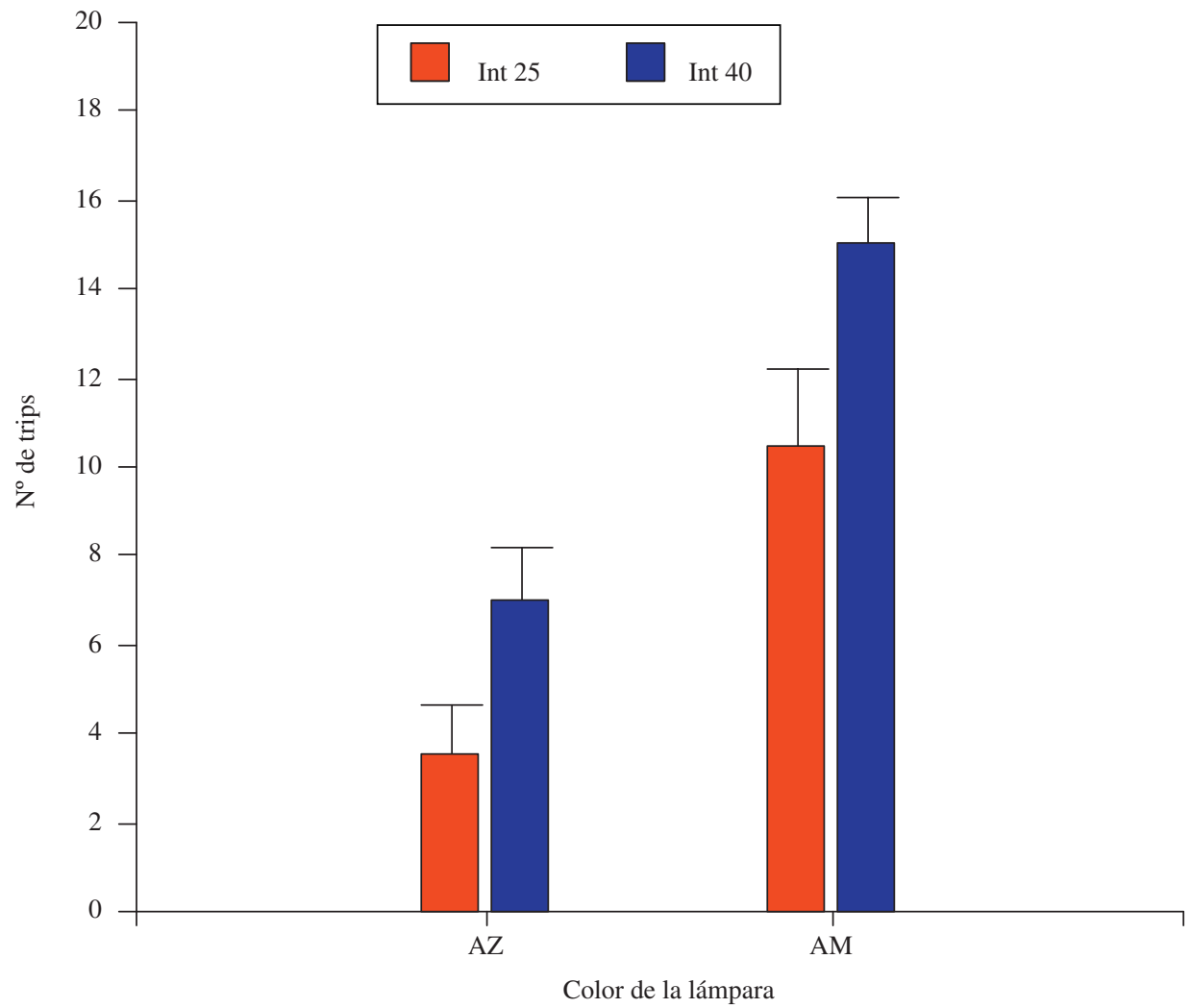

Figura 1: Efecto del color e intensidad de lámparas en trampas empleadas para la captura de trips. Promedios y error estándar de tratamientos. Todas las medias difieren entre sí (Test Tukey, alfa $=0,05$ ).

significativo en la emisión de señales visuales (color e intensidad) que modificarían el comportamiento de búsqueda de $F$. occidentalis hacia su fuente de alimentación, así como también para su oviposición (Moffitt, 1964). Esto puede explicar la preferencia de color de $F$. occidentalis en las investigaciones a campo hacia el blanco (Yudin et al., 1987) o el color amarillo (Cho et al., 1995). Mientras que en invernáculos $F$. occidentalis es más atraído por trampas azules (Gillespie y Vernon, 1990).

\section{LITERATURA CITADA}

ALLEN, W.R. \& J.A. MATTEONI. 1988. Cyclamen ringspot: epidemics in Ontario greenhouses caused by the tomato spotted wilt virus. Canadian Journal of Plant Pathology 10: 41-46.

BADO, S.G. Y VALVARROSA, A. 1999. "Plagas del cultivo de Gerbera bajo invernáculo". Revista Produciendo $\mathrm{N}^{\circ}$ 51. Pp. 60-64.

BRØDSGAARD, H.F. 1989. Coloured sticky traps for Frankliniella occidentalis (Pergande) (Thysanoptera: Thripidae) in glasshouses. Journal of applied entomology
Por lo tanto, estos ensayos muestran la necesidad de seguir investigando con diferentes tipos de ensayos la posibilidad de utilizar esta herramienta no sólo como método de monitoreo de la población de trips, para el uso más eficiente en la aplicación de insecticidas sintéticos, sino como un método de control para evitar que esta plaga llegue al UDE (Umbral de Daño Económico), evitando la aplicación de productos químicos que producen daño tanto al cultivo como a la fauna benéfica.

107: 136 -140. 1990: The effect of anisaldehyde as a scent attractant for Franklieniella occidentalis (Thysanoptera: Thripidae) and the response mechanism involved. Pp. 36-38. In: Brødsgaard, H.; Bennison, J.; Van Lenteren, J. C., (Eds). Working Group integrated control in Glasshouse Contributions to the meeting at Copenhagen (Denmark), 5-8 June 1990. SROP/WPRS Bulletin XIII/5.

BRØDSGAARD, H.F. 1993. Monitoring thrips glasshouse pot plant by means of blue sticky traps. Bulletin of OILB/ SROP 16: 29-32. 
BRYAN, D.E. AND SMITH, R.F. 1956. The Frankliniella occidentalis (Pergande) complex in California (Thysanoptera: Thripidae). University of California Publications in Entomology, 10 (6): 359-410.

CABELLO, T.; M.M.ABAD \& F. PASCUAL. 1991. Catches of $F$. occidentalis in traps of distinct colour in protect crops. Boletin de Sanidad Vegetal. Plagas 17: 265-270.

CARRIZO, P.I. 1998. Eficiencia de capturas con trampas de Frankliniella occidentalis (Pergande) en el cultivo de pimiento en invernáculo y en malezas en el Gran La Plata. Revista de la Facultad de Agronomía, La Plata 103 (1): 1-10.

CZENZ, K., 1987. The role of coloured traps in collecting thrips fauna. Pp. 426-435. In: Holman, J. Pelikán, J.; Dixon, A. F. G.; Weisman, L., (Eds). Population estructure, genetics and taxonomy of aphids and Thysanoptera. Proccedings of Internacional Symposia, held at Smolenice, Czechoslovakia, September 9-14, 1985. SPB Academia Publishing.

CHO, K.; ECKEL, C.S.; WALGENBACH, J.F. \& KENNEDY, G.G. 1995. Overwintering of thrips (Thysanoptera: Thripidae) in North Carolina. Environ. Entomol., 24: 58-67.

DE SANTIS, L. 1995. La presencia en la República Argentina del trips californiano de las flores, Frankliniella occidentalis (Pergande). Anales Academia Nacional de Agronomía y Veterinaria 49: 7-18.

EPPO. 1989. EPPO data sheets on quarantine organisms. EPPO Bulletin 19: 725-731.

FAEGRI, K.; VAN DE PIJL, L. 1979. The Principles of Pollination Ecology. 3 rd ed. Oxford, Pergamon Press. 244 pp.

GILLESPIE, D.R.; VERNON, R.S. 1990. Trap of catch of western flower thrips (Thysanoptera: Thripidae) as affected by color and height of sticky card traps in mature greenhouse cucumber crops. J. Econ. Entomol. 83, 971-975.
KIRK, W.D.J. 1984. Ecologically selective coloured traps. Ecol. Entomol. 9, 35-41.

LEWIS, T. 1959. A comparison a water traps, cylindrical sticky traps and suction traps for sampling thysanopteran populations at different levels. Entomologia experimentalis et applicata. 2: 204-215.

MATTESON, N.A. \& L.J. TERRY. 1992. Spectral efficiency of the western thrips flower, F. occidentalis. Journal of insect physiology 38: 453-459.

DE SANTIS, L. 1995. La presencia en La República Argentina del trips californiano de las flores, Frankliniella occidentalis (Pergande). Anales Academia Nacional de Agronomía y Veterinaria 49: 7-18.

MOFFIT, H.R. 1964. A color preference of the Western Flower Thrips, Frankliniella occidentalis. J. Econ. Entomol., 57: 604-605.

KАTАYАМА, H. 1997. Effect of temperature on development and oviposition of western flower thrips Frankliniella occidentalis (Pergande). Japanese Journal of applied Entomology and Zoology, 41: 225- 231

SHIPP, J.L.; ZARIFFA, N. 1990. Developing a sampling program for western flower thrips on greenhouses peppers. Pp. 194-197. In Brødsgaard, H.; Bennison, J.; Van Lenteren, J. C., (Eds). Working Group Integrated control in glasshouses Contribution to the meeting at Copenhagen (Denmark), 5-8 June 1990. SROP/WPRS Bulletin XIII/5.

VISSER, J.H. 1986. Host odour perception in phytophagous insect. Anual review of entomology 31: 121-144.

YUDIN, L.S.; W.G. MITCHELL \& J. J. CHO. 1987. Color preference of thrips (Thysanoptera: Thripidae) with reference to aphids (Homoptera: Aphididae) and leafminer in hawaiian lettuce farms. Journal of Economic Entomology 80: $51-55$ 